\title{
Análisis del modelo docente universitario y el uso de metodologías de enseñanza, aprendizaje en la evaluación de asignaturas de la praxis profesional en carreras de Gastronomía
}

\begin{abstract}
Analysis of the university teaching model and the use of teaching and learning methodologies for the assessment of professional training assignments in gastronomy undergraduate programs.
\end{abstract}

Inés Mariana Marín Parra. ${ }^{1}$, David Rodolfo Guambi Espinosa. ${ }^{2} \&$ Martha Cecilia Ávalos Pérez. ${ }^{3}$

Abstract. DOI: https://doi.org/10.33262/concienciadigital.v4i1.2.1591

Introduction. In the experiments carried out, a metric from 1 to 10 is used as a reference, with 1 being determined as the maximum deficient range and 10 as the maximum efficient range, this scale being commonly used in high education institutions. In each experiment, the different components of the aforementioned methodology for the practical subjects are integrated in order to assess the incidence of each of them in the student's receptivity to knowledge and to understand the relevance of the applied teaching methodology. Objective. To analyze the university teaching model and the use of teaching and learning methodologies for the assessment of professional training activities. Methodology. The research design begins with a descriptive-correlational study in which the behavior of the different variables and the incidence between them is analyzed. The research designs. is based on a descriptive-correlational study in which the behavior of specific variables and the incidence among them is analyzed. Results. The results found that in experiment (A) using $100 \%$ of the time with the theoretical approach, values concentrated in $2 / 10$

1 Escuela Superior Politécnica de Chimborazo, Facultad de Salud Pública. Riobamba, Ecuador. imarin@espoch.edu.ec, Orcid 0000-0001-7371-1100

2 Universidad UTE, Facultad de Ciencias Gastronómicas y Turismo. Quito, Ecuador. david.guambi@ute.edu.ec, Orcid 0000-0002-1667-5729

3 Escuela Superior Politécnica de Chimborazo, Facultad de Salud Pública. Riobamba, Ecuador. martha_avalos@espoch.edu.ec, Orcid 0000-0003-2519-3067 
(inefficient) are obtained. While in experiment (B) with $100 \%$ of the time of the lesson dedicated to practical activities, a concentration of 3/10 (inefficient) results are observed. In experiment $\mathrm{C}$, when incorporating an empirical activity, the concentration is situated in the range of 5/10 (inefficient). On the contrary, in experiment $\mathrm{D}$, when incorporating feedback within the time of the course, we find a result clustering around 6-7/10 (Acceptable). In comparison, in experiment $\mathrm{E}$, by adding a theoretical activity as a reinforcement task, we obtain results in the range of $6 / 10$ (Inefficient). Finally, tasks with practical activities with theoretical foundations are incorporated reflecting values around 7 and 8/10 (Acceptable). Conclusions. It is concluded that the implementation of each of the factors (theoretical induction, culinary practice, experiential activity, feedback, practical activities - theoretical) have a direct impact on student learning, understanding and receptivity, all directly influencing the assessments carried out.

Keywords: Pedagogical cycle, Culinary Practice, Experiential activity, Pedagogical feedback, Factors.

\section{Resumen.}

Introducción. En los experimentos realizados se toma como referencia una métrica de 1 al 10, determinándose como 1 el rango máximo deficiente y 10 como rango máximo eficiente, escala frecuentemente utilizada en instituciones de educación superior. En cada ensayo se incorpora los diferentes componentes de la metodología citada para las asignaturas prácticas a fin de observar la incidencia de cada uno de ellos en la recepción del conocimiento en el estudiante y entender la importancia de la metodología de enseñanza aplicada. Objetivo. Analizar el modelo docente universitario y el uso de metodologías de enseñanza aprendizaje, en el proceso de evaluación de asignaturas de la praxis profesional en carreras de Gastronomía. Metodología. El diseño de la investigación comienza mediante estudios descriptivos - correlacional en la que se analiza los comportamientos de la diferentes variables y la incidencia entre ellas. Resultados. Los resultados encontrados en el experimento (A) utilizando el 100\% del tiempo con el componente teórico se obtiene valores que se concentran en 2/10 (ineficiente). En el experimento (B) con el $100 \%$ del tiempo de la asignatura en procesos prácticos existe concentración de los resultados en 3/10 (ineficiente). En el experimento $\mathrm{C}$ al incorporar una actividad experiencial, la está alrededor de 5/10 (ineficiente). En el experimento D al incorporar retroalimentación en el tiempo de la asignatura observamos que se concentran 6-7/10 (Aceptable). En el experimento E se procede a adicionar una actividad teórica como tarea de refuerzo con lo que se obtiene resultados alrededor de 6/10 (Ineficiente). Finalmente se incorpora tareas con actividades prácticas con fundamentos teóricos lo que refleja valores alrededor de 7 y 8/10 (aceptable). Conclusiones. Se concluye que la aplicación de cada uno de los factores (inducción teórica, práctica culinaria, actividad experiencial, retroalimentación, tareas práctica - teóricas) tienen incidencia directa en el 
aprendizaje, comprensión y retención del estudiante con incidencia directa en las evaluaciones realizadas.

Palabras claves: Ciclo pedagógico, Práctica Culinaria, Actividad Experiencial, Retroalimentación pedagógica, Factores.

\section{Introducción.}

La formación gastronómica académica universitaria ha tomado presencia en el país ofertándose títulos de tercer nivel en universidades públicas y privadas, con significativa aceptación en los bachilleres ecuatorianos.

Desde el año 2000 aproximadamente la formación gastronómica se formalizó transformando en sí, la óptica de la profesión culinaria que hasta entonces era una ocupación empírica en la industria de alimentos y bebidas, en la cual este operario era carente de formación especializada, académica y profesional; realidad que a la actualidad ha evolucionado considerablemente debido a que la educación culinaria se hace presente en diferentes instituciones tecnológicas y universitarias como carreras terminales de tercer nivel, con proyección a estudios de cuarto nivel.

La formación gastronómica universitaria a diferencia de instituciones superiores tecnológicas oferta una educación integral en sus procesos (operativa, administrativa y de gestión) lo cual cambia la realidad de hace algunos años transformando al ejecutor empírico en un profesional titulado de tercer nivel con competencias integrales que responden a perfiles competitivos para involucramientos eficientes en jerarquías de dirección, gestión, administración con toma de decisiones empresariales basadas en su formación académica.

La reforma educativa actual enfatiza los procedimientos, valores y actitudes que constituyen el currículo y la intervención educativa. Según este lenguaje e impulso, el trabajo en grupo, la formación de equipos de aprendizaje, el desarrollo de actitudes de cooperación, etc., constituyen una variable importante de formación básica en la enseñanza superior, de cara a una eficaz integración de los egresados en el ámbito laboral (Gonzales y García, 2007). De allí la importancia en determinar la metodología de enseñanza aprendizaje adecuada para lograr una adecuada receptividad de los fundamentos teóricos en estas materias y con ello alcanzar que los procesos prácticos sean elaborados integralmente y se conviertan en procesos teóricos - prácticos.

Como afirma Ibarrola-García (2010), el profesor construye su conocimiento práctico cuando lo acepta como una característica básica de su profesión. También si ha aprendido como activar los procesos de reflexión que le permiten establecer sinergias entre el saber y el hacer. Mientras que el trabajar en pro del desarrollo del conocimiento en todas sus 
formas y expresiones y promover su utilización en todos los campos es una tarea que involucra e insta al compromiso de todos y cada uno de los profesionales académicos universitarios (Lira, R.2010). En la metodología utilizada para un proceso de enseñanza - aprendizaje intervienen muchas técnicas, instrumentos, actividades que dependen de los involucrados, pero la dinámica cae directamente sobre el instructor quien hará uso de sus habilidades y experiencia docente y profesional afín al conocimiento de la asignatura, manteniendo una relación directa entre el saber y el hacer en las asignaturas con procesos prácticos y tangibles.

A diferencia de otros campos profesionales, en donde la teoría es incuestionable como base para la actividad que desarrollan quienes ejercen la práctica, en el campo de la educación, la teoría y la práctica no siempre han ido de la mano. En general, la "buena práctica" es aquella que se apega a la teoría que ha sido reconocida y aceptada abiertamente por los miembros del colectivo que la ejercen, mientras que la práctica que se aleja de esta teoría corre el riesgo de ser no reconocida y, además, sancionada (Rodríguez y Alamilla, 2018). En este contexto la teoría y la práctica deben mantener una relación dinámica para conseguir procesos que garanticen confiabilidad. También es necesario involucrar al alumno como receptor de esta experiencia. El estudiante es el centro de esta enseñanza, su trabajo y su seguimiento son la base para su aprendizaje. Nos sorprende que algunas disciplinas en algunas universidades latinoamericanas desconozcan el papel de la tutoría y la evaluación (Barros, Chavarría y Paredes, 2008). Con la buena práctica metodológica aplicada por el docente se busca una educación integral que tenga un efecto multiplicador en la sociedad, aportando con soluciones a problemáticas que se adapten en el tiempo. Frente a los numerosos desafíos del porvenir, la educación constituye un instrumento indispensable para que la humanidad pueda progresar hacia los ideales de paz, libertad y justicia social (Delors, 1986).

El conocimiento impartido debe tener un objetivo de ejecución individual y colectivo como menciona Cano Ma. Elena (2008), es necesario ser capaz de buscar la información pertinente a cada momento, ser capaz de seleccionarla (de entre un abanico vastísimo de posibilidades), ser capaz de procesarla, tratarla, interpretarla y apropiarse de ella para generar el conocimiento necesario que nos permita resolver las situaciones que se nos presenten. Tal afirmación argumenta la importancia del conocimiento desarrollado en las aulas como protagonista de planteamientos que generen cambios en la colectividad que se apliquen, dando fiel cumplimiento a lo que dicta una nueva Ley Orgánica de Educación Superior de Ecuador que contribuya a la transformación de la sociedad, a su estructura social, productiva y ambiental, formando profesionales y académicos con capacidades y conocimientos que respondan a las necesidades del desarrollo nacional y a la construcción de ciudadanía (LOES, 2018).

Entonces si existe una normativa en la que declara que las instituciones de educación superior deben generar transformación en la sociedad es importante implicar el concepto de calidad pedagógica en los procesos de enseñanza para garantizar tal afirmación, según lo señala Garduño, L (1999), la calidad de la educación implica un proceso sistemático y 
continuo de mejora sobre todos y cada uno de sus elementos. Este compromiso con el mejoramiento viene dado por el propósito de la educación. Es vital generar condiciones que generen un mejoramiento continuo y aseguren la comprensión, análisis y desarrollo del conocimiento.

Las asignaturas practicas profesionalizantes usualmente tienen las características de atribuirse un número superior de horas en las diferentes mallas curriculares de las Instituciones de educación superior, por lo que la distribución del tiempo para cada componente pedagógico de cada sesión es de suma importancia para garantizar el aprendizaje en el educando. Según lo afirma Himmel, E. (2001), se puede señalar además que, durante el proceso de aprendizaje, la atención se mueve libremente entre los aspectos direccionales, algorítmicos y evaluativos. Asimismo, la cantidad de tiempo dedicado a cada componente depende principalmente de la naturaleza del objetivo, el estilo de aprendizaje del sujeto, y su nivel de desarrollo. La riqueza, profundidad y variabilidad del conocimiento y experiencia docente es importante para identificar las mejores estrategias empleadas en el curso del ciclo pedagógico utilizado.

Para el análisis de la influencia de los factores que intervienen en el ciclo pedagógico y los resultados reflejados en las evaluaciones realizadas a los estudiantes se toma como referencia dos instituciones universitarias del país da las cuales se recopilan los siguientes datos:

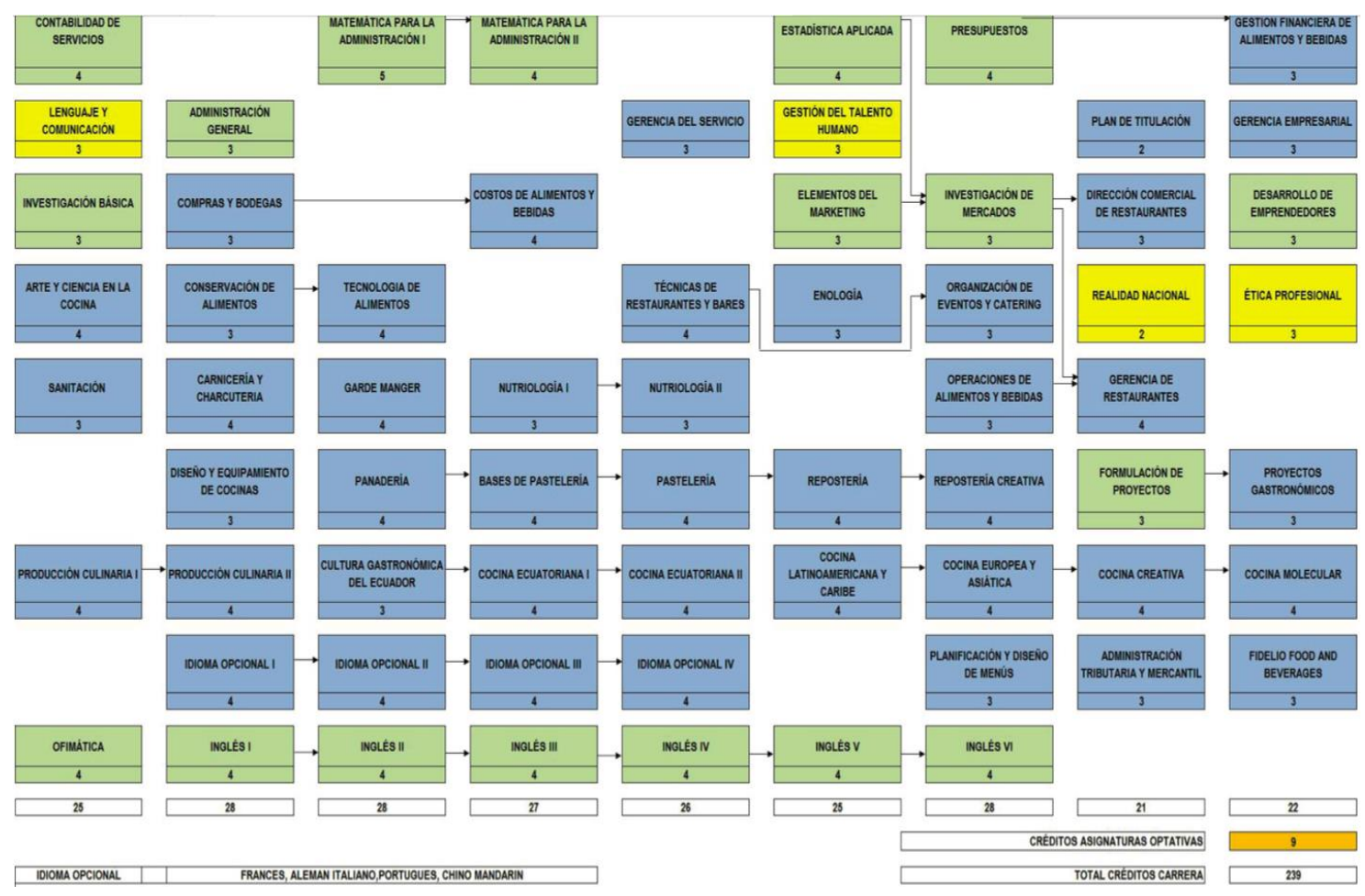

Figura 1. Malla curricular Carrera de ciencias gastronómicas de la Universidad UTE.

Fuente: www.ute.edu.ec 
ISSN: 2600-5859

Tabla 1. Asignaturas prácticas profesionalizantes que componen la malla curricular de la carrera de gastronomía de la Universidad UTE

\begin{tabular}{|c|l|}
\hline \multicolumn{2}{|c|}{ ASIGNATURAS PRÁCTICAS PROFESIONALIZANTES } \\
\hline No & \multicolumn{1}{|c|}{ ASIGNATURA } \\
\hline 1 & Técnicas Básicas de cocina I \\
\hline 2 & Técnicas Básicas de cocina II \\
\hline 3 & Técnicas de Carniceria y Charcuteria \\
\hline 4 & Panaderia \\
\hline 5 & Garde Manger \\
\hline 6 & Servicio de Bebidas \\
\hline 7 & Técnicas Básicas de Pastelería \\
\hline 8 & Cocina Europea Asiatica \\
\hline 9 & Pastelería Clásica \\
\hline 10 & Cocina Latinoamericana y El Caribe \\
\hline 11 & Repostería \\
\hline 12 & Cocina regional Sierra y Galápagos \\
\hline 13 & Chocolateria y confiteria ecuatoriana \\
\hline 14 & Cocina Regional costa y oriente \\
\hline 15 & Gastronomía aplicada en Alimentos y Bebidas \\
\hline 16 & Repostería Creativa \\
\hline 17 & Cocina de vanguardia \\
\hline
\end{tabular}

Fuente: www.ute.edu.ec

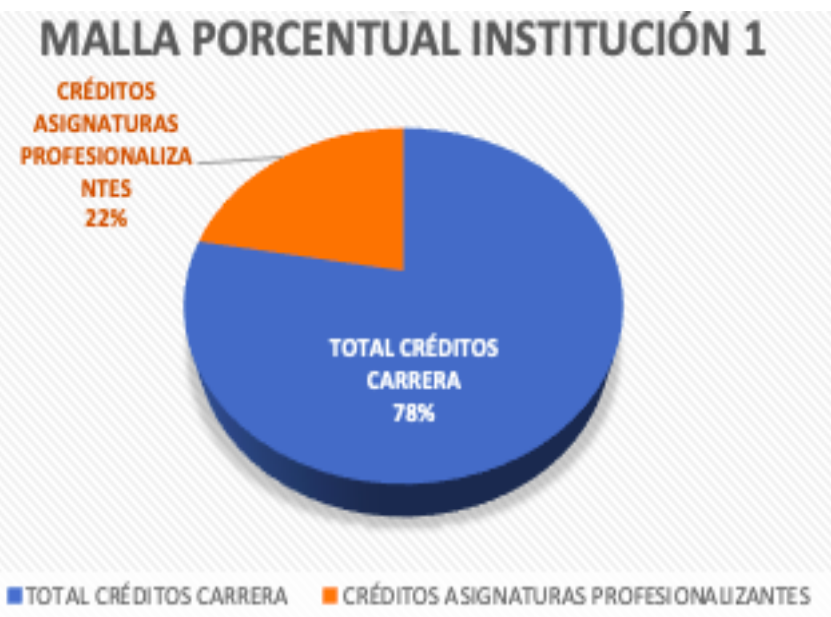

Figura 2. Valor porcentual que ocupa las materias prácticas profesionalizantes versus el total de materias de la malla curricular carrera Ciencias Gastronómicas UTE.

Fuente: Elaboración propia.

Total, créditos de la carrera: 239

Asignaturas prácticas profesionalizantes: 17

Créditos asignaturas profesionalizantes: 68 


\section{MALLA CURRICULAR INSTITUCIÓN 2}

Tabla 2. Malla curricular carrera de ciencias gastronómicas de la Escuela Politécnica de Chimborazo.

\begin{tabular}{|c|c|c|c|c|c|c|c|}
\multicolumn{7}{c|}{ CUADRO RESUMEN DE ESTRUCTURA CURRICULAR DE LA CARRERA GASTRONOMIA } \\
\hline $\begin{array}{c}\text { NO. } \\
\text { ASIGNATURAS }\end{array}$ & $\begin{array}{c}\text { TOTAL } \\
\text { CRÉDITOS }\end{array}$ & TOTAL HORAS DE LA CARRERA & $\begin{array}{c}\text { HORAS DE } \\
\text { APRENDIZAIE EN } \\
\text { CONTACTO CON EL } \\
\text { DOCENTE }\end{array}$ & $\begin{array}{c}\text { HORAS DE APRENDIZAIE } \\
\text { PRÁCTICO EXPERIMENTAL }\end{array}$ & $\begin{array}{c}\text { HORAS DE } \\
\text { APRENDIZAIE } \\
\text { AUTÓNOMO }\end{array}$ & $\begin{array}{c}\text { HOTALES DE } \\
\text { PRÁCTICAS } \\
\text { PREPROFESION } \\
\text { ALES }\end{array}$ & $\begin{array}{c}\text { HORAS DE INTEGRACIÓN } \\
\text { CURRICULAR }\end{array}$ \\
\hline 49 & 120 & 5760 & 1936 & 2000 & 1824 & 480 \\
\hline
\end{tabular}

Fuente: www.espoch.edu.ec

Tabla 3. Asignaturas prácticas profesionalizantes que componen la malla curricular de la Carrera de Gastronomía de la Escuela Superior Politécnica de Chimborazo

\begin{tabular}{|c|l|}
\hline \multicolumn{2}{|c|}{ ASIGNATURAS PRÁCTICAS PROFESIONALIZANTES } \\
\hline № & \multicolumn{1}{c|}{ ASIGNATURA } \\
\hline 1 & Técnicas I \\
\hline 2 & Procesamiento de alimentos \\
\hline 3 & Técnica de servicio de restuarantes \\
\hline 4 & Procesamiento de alimentos en panaderia \\
\hline 5 & Procesamiento de alimentos cárnicos \\
\hline 6 & Gastronomía Internacional \\
\hline 7 & Procesamiento de alimentos pasteleria y reposteria \\
\hline 8 & Gastronomía Internacional América \\
\hline 9 & Gastronomia Ecuatoriana región costa \\
\hline 10 & Arte y modelaje de alimentos \\
\hline 11 & Gastronomia ecuatoriana región amazónica \\
\hline 12 & Gastronomía Internacional Europa \\
\hline 13 & Gastronomía nutricional y hospitalaria \\
\hline 14 & Gastronomía institucional y banquetes \\
\hline 15 & Enologia y mixiología \\
\hline 16 & Gastronomía de vanguardia I \\
\hline 17 & Gastronomía Ecuatoriana región sierra \\
\hline 18 & Gastronomía de vanguardia II \\
\hline
\end{tabular}

Fuente: www.espoch.edu.ec

Cada asignatura práctica profesionalizante tiene una carga de 8 horas semanales, con un total semanal de 136 horas.

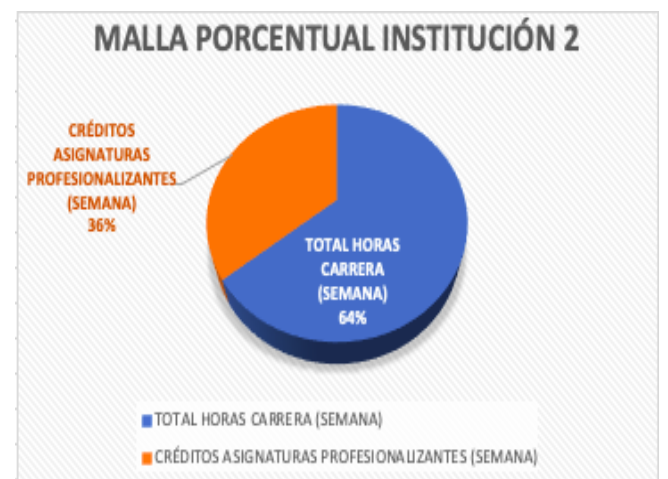

Figura 3. Valor porcentual que ocupa las materias prácticas profesionalizantes versus el total de materias de la malla curricular escuela de gastronomía ESPOCH. Elaboración propia. 
La formación gastronómica como carrera universitaria forma parte del área de servicios, por ende, la formación en las asignaturas señaladas implica procesos prácticos en laboratorios por lo que el análisis de la metodología de enseñanza - aprendizaje se desarrollará exclusivamente en estas asignaturas para identificar la incidencia de los factores empleados en los resultados cuantitativos en las evaluaciones.

\section{Proceso culinario profesional}

Lo más frecuente es que la relación alumno-contenido se produzca a través de algún medio, material o recurso didáctico que represente, aproxime o facilite el acceso del alumno a la observación, investigación o comprensión de la realidad (Romero, Amaya y Castaña, 2020). Por lo que es necesario que el ejercicio experiencial aplicado en cada sesión de las asignaturas prácticas manifieste procesos que usualmente se aplica en un proceso culinario utilizado en la industria de Alimentos y bebidas, con el fin de que el estudiante adquiera competencias adecuadas para su desenvolvimiento adecuado en su rol laboral. Según Roig, Lledo, Antolí y Pellín (2019), el alumno como futuro profesional de la gastronomía necesita familiarizarse con un lenguaje que permita expresar ideas, conceptos y realidades característicos del mundo de la gastronomía y la cocina. De hecho, es necesario disponer de habilidades en cálculos básicos para desarrollar recetas de cocina y evaluar y comprender críticamente tablas de composición de alimentos, así como desarrollar informes correctamente y presentar habilidades informáticas y de idioma extranjero, entre otras.

El ciclo de enseñanza en una materia práctica profesionalizante inicia con el abastecimiento de la documentación necesaria a los estudiantes, los mismos que son en base a formatos institucionales estandarizados donde consta de menús, recetas, requisiciones, órdenes de compra de la materia prima necesaria para la ejecución de los procedimientos culinarios planificados en los laboratorios. Este proceso con el fin de dar cumplimiento al acercamiento de un proceso real como se había mencionado. Estas intentan garantizar la calidad, seguridad e inocuidad de las comidas por medio de métodos que incluyen la producción, manipulación, transporte, almacenamiento y/o distribución de los alimentos. Los factores como la calidad de la materia prima, las condiciones ambientales, las características de los equipamientos usados en la preparación y las condiciones técnicas de higiene son fundamentales para la prevención de Enfermedades Transmitidas por los Alimentos (ETA) provenientes de bacterias, virus y parásitos, (Correia, Araújo, Fernández, Leao y Pinheiro, 2012). 


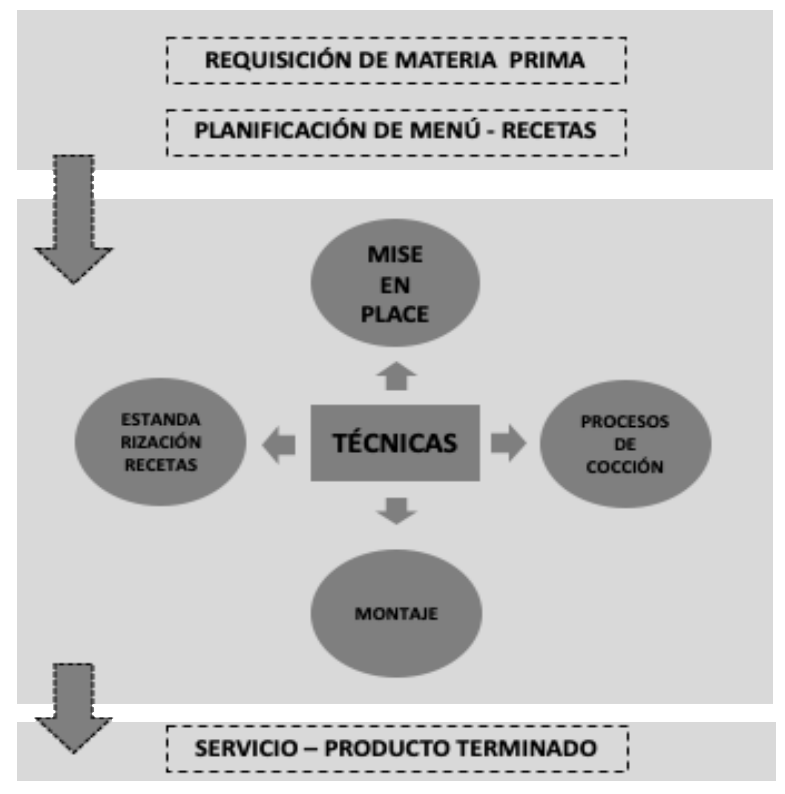

Figura 4. Proceso culinario aplicado en las materias prácticas.

Fuente: Elaboración propia.

El proceso de enseñanza de una sesión en las asignaturas prácticas inicia con la planificación de las recetas y técnicas a realizarse, de las mismas que se obtiene la documentación necesaria desarrollada mediante formatos para cada etapa de la gestión de compra como son las recetas y requisiciones de materia prima así, consecuente es la elaboración del mise en place, término utilizado al proceso de limpieza, cortes y picados de la materia prima a emplearse, así como el pesaje exacto y orden obligatorio indicado por la receta estándar. Esta etapa será determinante en la productividad adecuada en el operario (estudiante) para la aplicación de los procesos culinarios empleándose técnicas profesionales que al terminar facilitaran el montaje de un plato con estándares técnicos, profesionales con la teoría implícita en cada etapa de elaboración donde como resultado un producto culinario final.

\section{Factores metodológicos citados en las materias prácticas}

En todo plan didáctico deben considerarse los siguientes elementos: competencias, contenidos, actividades, técnicas de enseñanza, recursos auxiliares e instrumentos de evaluación (Cifuentes, I. 2012). 


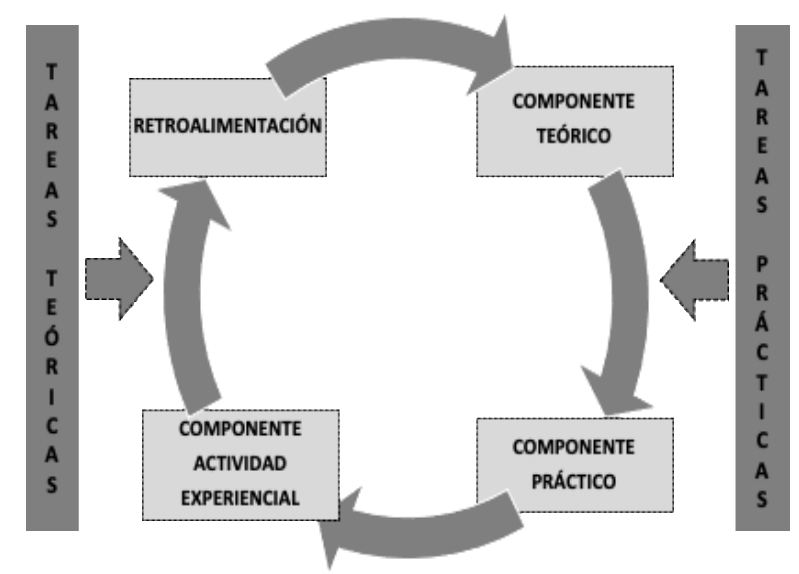

Figura 5. Ciclo Pedagógico de enseñanza - aprendizaje aplicado en la investigación.

Fuente: Elaboración propia.

Todos estos factores intervienen en el ciclo de enseñanza pedagógica a fin de obtener la transferencia de conocimientos adecuados, tratándose con ello de alcanzar resultados óptimos de conocimiento teórico prácticos en el estudiante, con el fin de alcanzar calidad educativa en el estudiantado.

Al finalizar ciclos académicos, se determina en la evaluación que existe un desfase entre el conocimiento teórico y el práctico, observándose que los procesos desarrollados por los estudiantes en las horas asignadas para esta actividad tienen una gran dosis de empirismo, criterio personal y falta de interpretación teórica en sus procesos gastronómicos.

En los procesos de enseñanza y comprensión es fundamental todo lo que tenga que ver con las prácticas pedagógicas, es decir toda las actividades que se van a volver acciones en el aula de clase y que ayudan a la interacción docente-alumno, con el fin de que haya un mejor entendimiento de los temas; es decir, que el estudiante vaya construyendo su conocimiento y por ende su comprensión (Castillo, 2012).Todo esto detectado por el bajo nivel cuantificado en las evaluaciones teóricas realizadas en los alumnos.

De allí nace la necesidad de realizar la investigación mediante un experimento en el cual se involucra paso a paso los factores componentes del ciclo pedagógico: Inducción Teórica; practica culinaria; actividad experiencial y retroalimentación. Determinando así el rol, importancia e influencia en cada una de ellas en el resultado final en el estudiante: una transferencia de conocimiento completo con resultados e interpretación adecuados, conocimientos prácticos teóricos.

Como eje principal para el posterior desarrollo profesional de los estudiantes se desarrolla el análisis en los alumnos de primer y segundo nivel en las materias profesionalizantes Producción Culinaria I y Producción Culinaria II, debido a que en ellas se transmite todos 
los conocimientos teórico - prácticos que serán replicados en las materias consecutivas a estas en los niveles superiores.

\section{Descripción}

El conocimiento profesionalizante en los estudiantes es un factor muy importante en los futuros Administradores Gastronómicos debido a que entre su perfil desarrolla destrezas adquiridas en las materias con metodología práctica teórica, las mismas que al ser evaluadas periódicamente, existe una deficiencia considerable en los conocimientos teóricos, los mismos q son muy escasos a la hora de ser evaluados, trayendo como consecuencia que los procesos culinarios sean empíricos, carentes de conocimiento científico - académico. De acuerdo con las observaciones en periodos pasados se observa una diferencia abismal entre las evaluaciones practicas con las teóricas, en un promedio de 8 a 4 respectivamente.

Razón por la que se pretende buscar la influencia de cada factor involucrado en el ciclo de enseñanza, a fin de determinar el nivel de importancia de cada uno de ellos en la salida (conocimiento teórico) en las materias prácticas.

\section{Experimento Ciclo Pedagógico en las materias practicas profesionalizantes gastronómicas.}

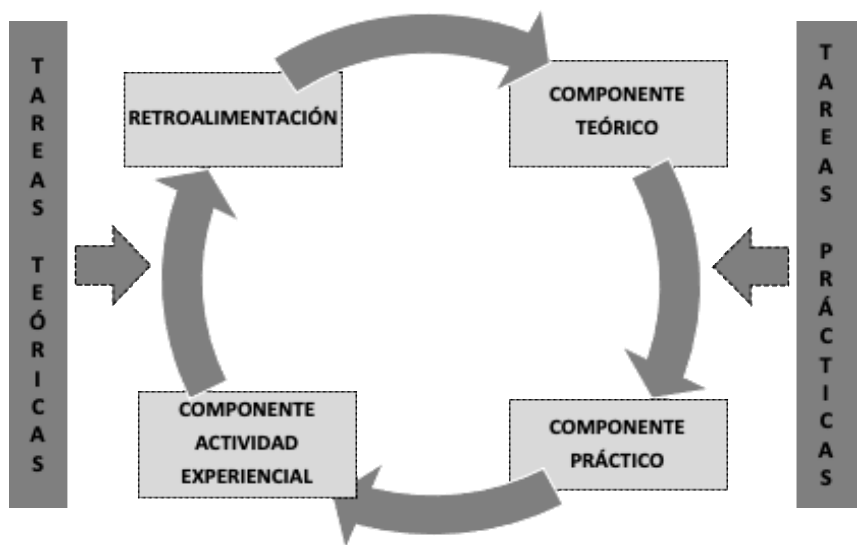

Figura 6. Esquema de la investigación a experimentarse.

Fuente: Elaboración propia.

El experimento por desarrollarse en cada tratamiento se realizará introduciendo paulatinamente cada factor, con el fin de observar la influencia en el nivel de aprendizaje teórico - practico en el estudiante (salida).

Hipótesis Nula: El rango de calificaciones es igual en las evaluaciones independientemente de los factores aplicados 
Hipótesis Alternativa: El rango de calificaciones es variable en las evaluaciones de acuerdo con los factores aplicados.

\section{Objetivo general}

Analizar el modelo docente universitario y el uso de metodologías de enseñanza aprendizaje, en el proceso de evaluación de asignaturas de la praxis profesional en carreras de Gastronomía.

\section{Objetivos específicos}

Identificar la influencia del factor en el resultado teórico práctico alcanzado en el estudiante luego de un proceso pedagógico gastronómico.

Determinar el nivel de importancia en cada factor para alcanzar rangos adecuados dentro de los límites aceptables.

\section{Metodologia.}

El diseño de la investigación comienza mediante estudios descriptivos - correlacional en la que se realiza una descripción de las metodologías pedagógicas conceptuales para luego realizar análisis de los comportamientos de las variables y la incidencia entre ellas mediante experimentos estadísticos de carácter cuantitativos.

Los métodos para obtener datos necesarios para la valoración del experimento fueron mediante evaluaciones teóricas luego de la aplicación de cada proceso realizado, con la adición de cada factor en los diferentes tratamientos aplicados.

Las evaluaciones teóricas fueron realizadas en el 100\% de los alumnos existentes en las asignaturas Producción Culinaria I y Producción Culinaria II, debido a que en estas cátedras se transmiten todo el conocimiento técnico base que serán replicados posteriormente en la praxis profesional de niveles superiores.

\section{Resultados.}

Tabla 4. Registro de notas alcanzadas por los estudiantes con la aplicación de diferentes factores que componen el ciclo pedagógico de enseñanza aprendizaje.

\begin{tabular}{ccccccc}
\hline \multicolumn{7}{c}{ DATOS RECOPILADOS EVALUACIONES DE ACUERDO A LOS ENSAYOS } \\
\hline \multirow{2}{*}{ No. } & A & B & C & D & E & F \\
& 1,5 & 3 & 2,5 & 2,5 & 8 & 5 \\
1 & 2 & 3 & 4 & 3,5 & 3 & 7,5 \\
2 & 3 & 4 & 3 & 6 & 3 & 7 \\
3 & & 7
\end{tabular}




\begin{tabular}{|c|c|c|c|c|c|c|}
\hline 4 & 2 & 3 & 4 & 4 & 4,5 & 7,5 \\
\hline 5 & 5,5 & 3 & 3,5 & 7 & 4,5 & 7 \\
\hline 6 & 5 & 3 & 4 & 6 & 5 & 6 \\
\hline 7 & 1 & 2 & 4,5 & 6,5 & 5 & 7,5 \\
\hline 8 & 2 & 2 & 2 & 7 & 4,5 & 7 \\
\hline 9 & 7 & 3 & 7 & 6 & 7,5 & 7,5 \\
\hline 10 & 1 & 0 & 4,5 & 3,5 & 7,5 & 6 \\
\hline 11 & 2 & 5 & 5 & 7 & 4 & 7,5 \\
\hline 12 & 5 & 3 & 5 & 3 & 4 & 6 \\
\hline 13 & 5 & 3 & 5 & 7 & 7 & 6 \\
\hline 14 & 2 & 2 & 4,5 & 7 & 4 & 7 \\
\hline 15 & 2 & 4 & 3 & 7 & 4 & 6 \\
\hline 16 & 1 & 4 & 3 & 7 & 7,5 & 7,5 \\
\hline 17 & 1 & 6 & 3 & 4,5 & 6 & 8,5 \\
\hline 18 & 4,5 & 3 & 5 & 3 & 6 & 7 \\
\hline 19 & 2 & 5 & 6 & 4,5 & 6,5 & 7 \\
\hline 20 & 2 & 3 & 6 & 7 & 6 & 5 \\
\hline 21 & 6 & 3 & 4,5 & 3 & 6 & 7,5 \\
\hline 22 & 4,5 & 3 & 5 & 5,5 & 6 & 7,5 \\
\hline 23 & 2 & 4 & 4,5 & 8 & 6,5 & 7,5 \\
\hline 24 & 0,5 & 3 & 4,5 & 6 & 5 & 6 \\
\hline 25 & 4 & 1 & 5 & 4,5 & 5,5 & 7,5 \\
\hline 26 & 5,5 & 3 & 5 & 6 & 5 & 7,5 \\
\hline 27 & 2 & 3 & 5 & 7 & 4 & 6 \\
\hline 28 & 3,5 & 4 & 5,5 & 5,5 & 4,5 & 6 \\
\hline 29 & 2 & 6 & 5 & 4,5 & 3 & 7 \\
\hline 30 & 2 & 2 & 5 & 7 & 3,5 & 6,5 \\
\hline 31 & 1,5 & 2 & 6 & 7 & 5,5 & 6,5 \\
\hline 32 & 1 & 2 & 6 & 5 & 5,5 & 6 \\
\hline 33 & 2,5 & 3 & 3 & 6 & 5,5 & 6 \\
\hline 34 & 2,5 & 5 & 3 & 6 & 5,5 & 6 \\
\hline 35 & 0,5 & 1 & 4 & 7 & 6 & 7,5 \\
\hline 36 & 1,5 & 4 & 2 & 3 & 6,5 & 7 \\
\hline 37 & 2 & 3 & 4,5 & 6 & 4 & 7,5 \\
\hline 38 & 2 & 4 & 4 & 6 & 6,5 & 6 \\
\hline 39 & 3,5 & 4 & 4 & 6 & 5 & 6 \\
\hline 40 & 2,5 & 3 & 4,5 & 6 & 5 & 7,5 \\
\hline 41 & 2 & 3 & 3 & 6 & 5 & 6 \\
\hline 42 & 2 & 1 & 5 & 6 & 5 & 6,5 \\
\hline 43 & 6 & 3 & 3 & 6 & 5 & 7,5 \\
\hline 44 & 5 & 3 & 6 & 7 & 6,5 & 7,5 \\
\hline 45 & 5 & 1 & 6,5 & 7,5 & 8 & 9 \\
\hline 46 & 4,5 & 3 & 9 & 8 & 7,5 & 9 \\
\hline 47 & 2 & 2 & 5 & 4 & 7,5 & 9,5 \\
\hline 48 & 5 & 2 & 4,5 & 8 & 3 & 8,5 \\
\hline 49 & 2 & 4 & 5 & 8,5 & 8 & 4,5 \\
\hline
\end{tabular}


ISSN: 2600-5859

\begin{tabular}{lcccccc}
\hline $\boldsymbol{5 0}$ & 4 & 3 & 5 & 8 & 4,5 & 5 \\
$\mathbf{5 1}$ & 3,5 & 3 & 3 & 3,5 & 5 & 5,5 \\
$\mathbf{5 2}$ & 2 & 4 & 3 & 6,5 & 5 & 4,5 \\
$\mathbf{5 3}$ & 9,5 & 4 & 2 & 5 & 5 & 7 \\
$\mathbf{5 4}$ & 7 & 4 & 5,5 & 6,5 & 5,5 & 7 \\
$\mathbf{5 5}$ & 5,5 & 5 & 1 & 5 & 4,5 & 7 \\
$\mathbf{5 6}$ & 2,5 & 5 & 4 & 6 & 4 & 7 \\
$\mathbf{5 7}$ & 4 & 1 & 4,5 & 6 & 7,5 & 7,5 \\
$\mathbf{5 8}$ & 9,5 & 3 & 4,5 & 6 & 8 & 4,5 \\
$\mathbf{5 9}$ & 2,5 & 1 & 4,5 & 6,5 & 8,5 & 6 \\
$\mathbf{6 0}$ & 9,5 & 3 & 6 & 7 & 10 & 4 \\
$\mathbf{6 1}$ & 2 & 3 & 6 & 6,5 & 5,5 & 4 \\
$\mathbf{6 2}$ & 6 & 0 & 6 & 7 & 4 & 3 \\
$\mathbf{6 3}$ & 6,5 & 2 & 5,5 & 6,5 & 4,5 & 6,5 \\
$\mathbf{6 4}$ & 7,5 & 3 & 7,5 & 7 & 5,5 & 4,5 \\
$\mathbf{6 5}$ & 1 & 3 & 2 & 5 & 4 & 5,5 \\
\hline
\end{tabular}

Fuente: Elaboración propia.

\section{En donde:}

\section{Factor A}

Corresponde a la aplicación del FACTOR A; Clase basada en el componente teórico con el $100 \%$ del tiempo asignado a las asignaturas profesionalizantes (4 horas).
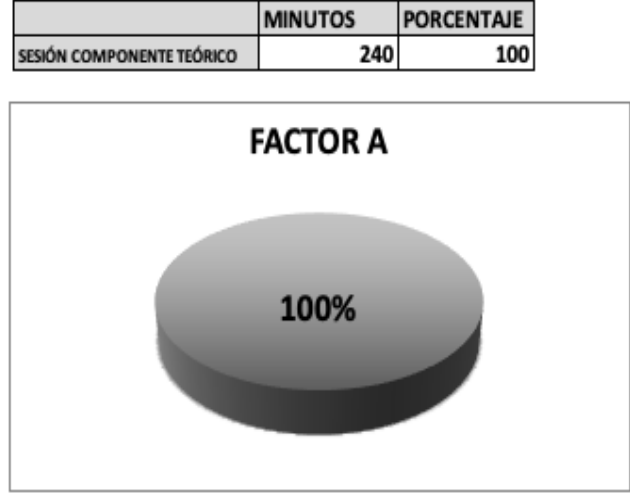

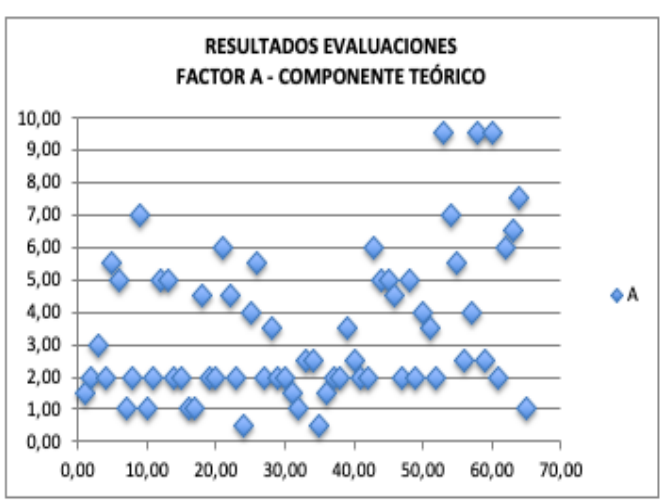

Figura 7. Gráficos porcentuales y dispersión de las notas alcanzadas con la metodología 100\% teórica.

Fuente: Elaboración propia.

Las notas alcanzadas en las evaluaciones están centradas a un promedio de 3,45, notas extremadamente inferiores al rango mínimo aceptable (7). 


\section{Factor B}

Corresponde a la aplicación del FACTOR B; Clase basada en el componente práctico con el $100 \%$ del tiempo asignado a las asignaturas profesionalizantes (4 horas).

\begin{tabular}{|l|r|r|}
\hline & MINUTOS & PORCENTAIE \\
\hline SESIÓN COMPONENTE PRACCTICO & 240 & 100 \\
\hline
\end{tabular}

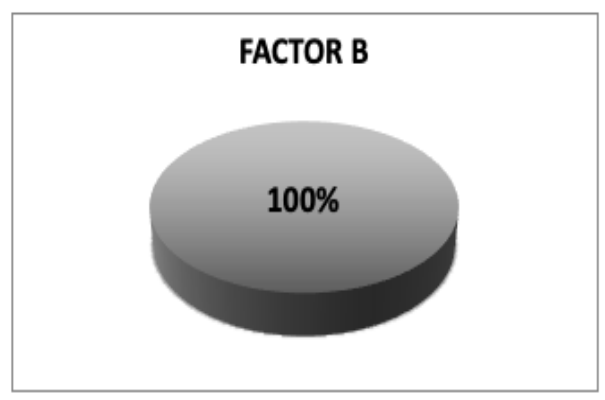

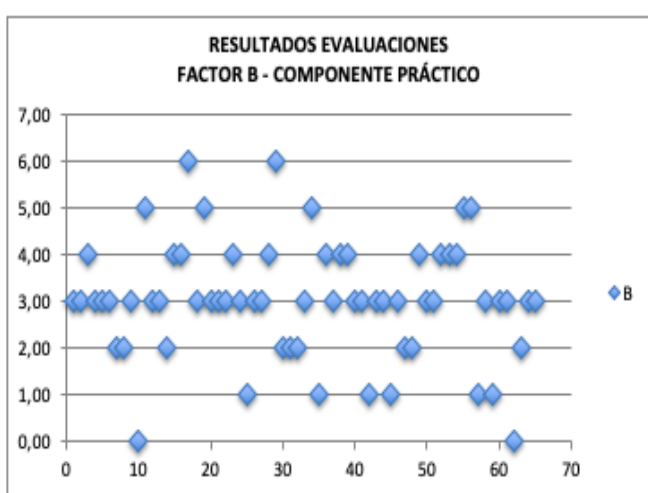

Figura 8. Gráficos porcentuales y dispersión de las notas alcanzadas con la metodología 100\% práctica.

Fuente: Elaboración propia.

Las notas alcanzadas en las evaluaciones se concentran en un promedio de 3,02 notas extremadamente inferiores al rango mínimo aceptable (7).

\section{Factor C}

Corresponde a la adición del FACTOR C; Actividad experiencial, en donde se determina 20 minutos del tiempo total asignado, para que los alumnos recopilen apuntes de los temas y técnicas culinarias realizados por el docente con una explicación detallada del proceso, interactuando con los alumnos y el tiempo para la recopilación de apuntes.

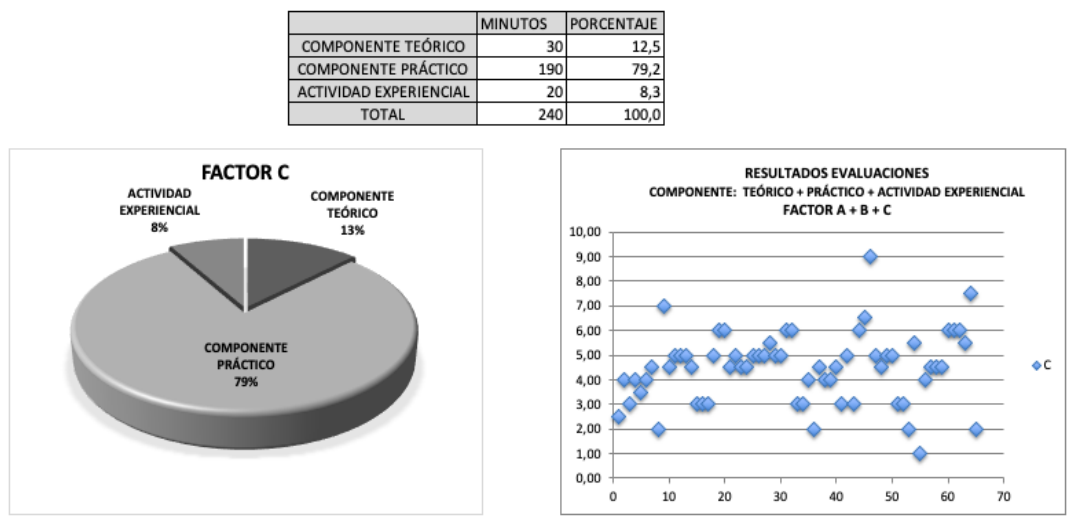

Figura 9. Gráficos porcentuales y dispersión de las notas alcanzadas con la introducción de factores actividad experiencial.

Fuente: Elaboración propia

Las notas alcanzadas en las evaluaciones se concentran en un promedio de 5 notas inferiores al rango mínimo aceptable (7). 


\section{Factor D}

Corresponde a la adición del FACTOR D; Retroalimentación, en donde se destina 30 minutos del tiempo total asignado, para la revisión, análisis, interacción con los estudiantes sobre los elementos teóricos, prácticos de los procesos realizados y los resultados obtenidos, y las posibles mejoras.

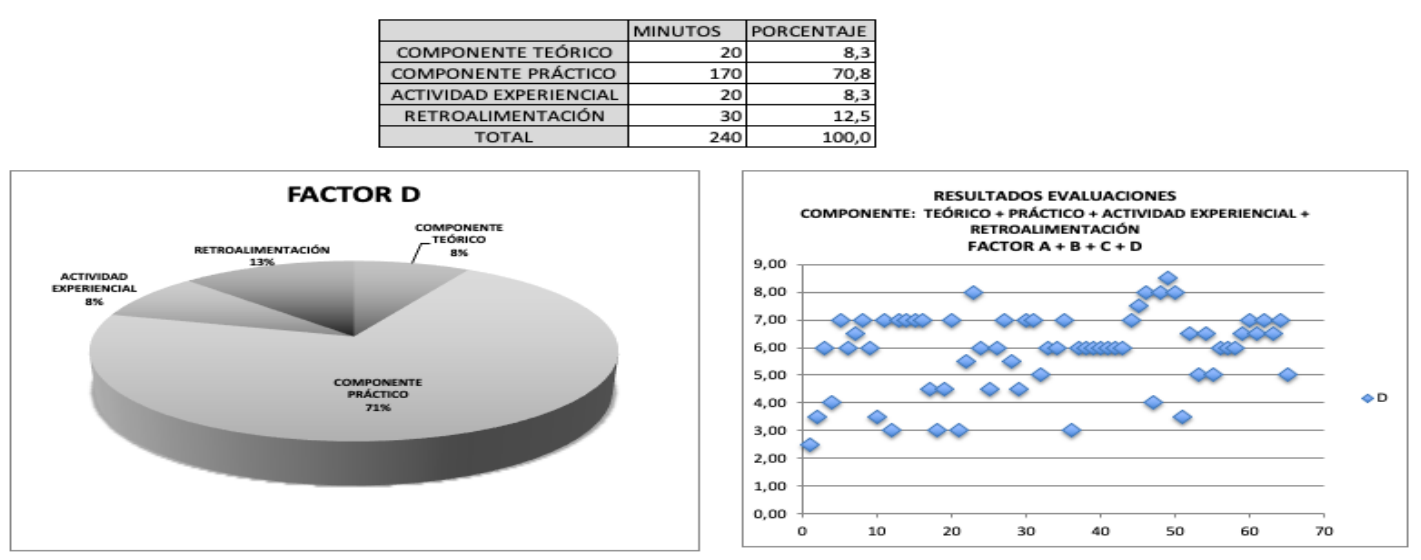

Figura 10. Gráficos porcentuales y dispersión de las notas alcanzadas con la introducción de factores actividad experiencial, retroalimentación.

Fuente: Elaboración propia.

Las notas alcanzadas en las evaluaciones se concentran en un promedio de 6 - 7 valores cercanas mínimo aceptable (7).

\section{Factor E}

Corresponde a la adición del FACTOR E; tareas escritas, teóricas como instrumento de refuerzo a los procesos prácticos planificados en la sesión de las materias profesionalizantes.

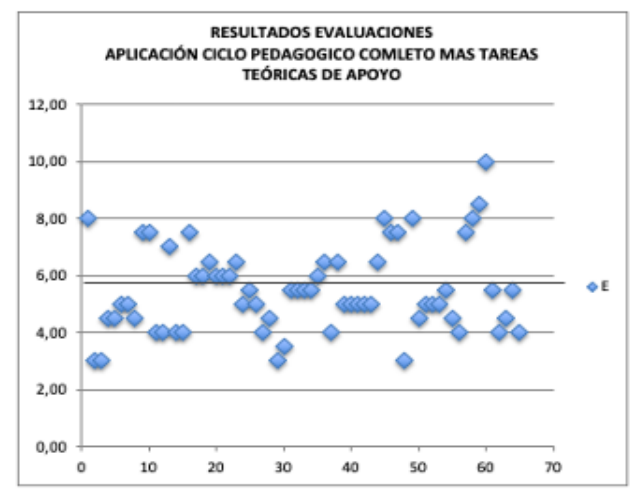

Figura 11. Porcentuales y dispersión de las notas alcanzadas con la introducción de factores actividad experiencial, retroalimentación, tareas teóricas.

Fuente: Elaboración propia.

Las notas alcanzadas en las evaluaciones se concentran en un promedio de 6 valores en inferiores al mínimo aceptable (7).

\section{Factor $\mathbf{F}$}

Corresponde a la adición del FACTOR F; Experimento realizado con el proceso metodológico completo, involucrando a todos los componentes que conforman el ciclo 
de enseñanza, en el que se utilizan tareas prácticas (videos) como instrumento de apoyo al aprendizaje.

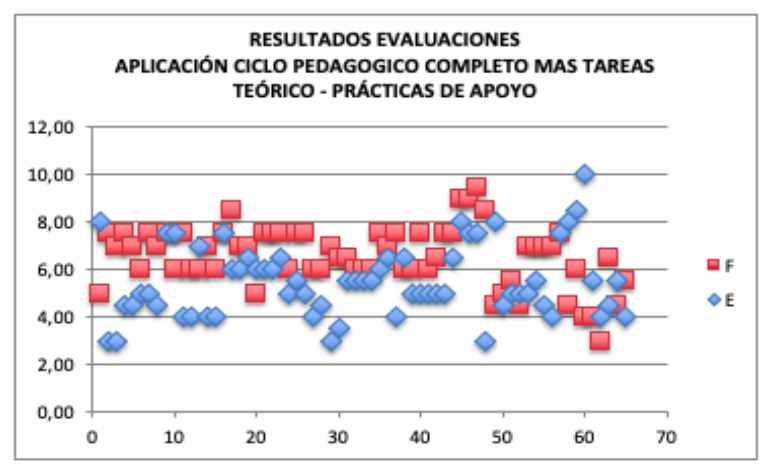

Figura 12. Gráficos porcentuales y dispersión de las notas alcanzadas con la introducción de factores actividad experiencial, retroalimentación, tareas prácticas.

Fuente: Elaboración propia.

Las notas alcanzadas en las evaluaciones se concentran en un promedio de 7 8valores dentro de los rangos aceptables (7 - 8).

Tabla 4. Prueba de hipótesis

\begin{tabular}{|l|r|r|r|r|r|r|}
\hline \multicolumn{1}{|c|}{ Resumen } & & & & & \\
\hline \hline Grupos & Tamaño muestral & Suma & Media & Varianza & & \\
\hline A & 65 & 212 & 3.26154 & 4.82115 & & \\
\hline C & 65 & 196 & 3.01538 & 1.57788 & & \\
\hline$D$ & 65 & 282 & 4.33846 & 2.00865 & & \\
\hline E & 65 & 374 & 5.75385 & 2.21971 & & \\
\hline F & 65 & 344 & 5.29231 & 2.17885 & & \\
\hline \hline & 65 & 412 & 6.33846 & 1.47740 & & \\
\hline ANOVA & & & & & & \\
\hline \hline Origen de la Variación & $S S$ & $d f$ & MS & $F$ & nivel p & F crit \\
\hline Entre Grupos & 596.51282 & 5119.30256 & 50.11430 & 0 & 2.23749 \\
\hline Dentro de Grupos & 914.15385 & 384 & 2.38061 & & & \\
\hline & & & & & & \\
\hline Total & $1,510.66667$ & 389 & & & & \\
\hline \hline
\end{tabular}

Fuente: Elaboración propia

\section{Discusión.}

La aplicación es de los factores que componen el ciclo pedagógico aplicado en las materias profesionalizantes, determina el grado de transmisión, retención y comprensión del conocimiento en los estudiantes, reflejándose en los valores alcanzados por los estudiantes en las diferentes evaluaciones.

Conforme se van añadiendo cada factor el rango de calificaciones tiene una variabilidad significativa. 


\section{Conclusiones.}

- Se concluye que la aplicación de cada uno de los factores (inducción teórica, práctica culinaria, actividad experiencial, retroalimentación, tareas práctica teóricas) tienen incidencia directa en el aprendizaje, comprensión y retención del estudiante con incidencia directa en las evaluaciones realizadas.

- Los resultados cuantificables en las evaluaciones teóricas dependen del ciclo pedagógico aplicado en cada clase práctica.

- Cada factor que intervino en los diferentes experimentos:

- Inducción teórica

- Práctica culinaria

- Actividad experiencial

- Retroalimentación

- Tareas prácticas

- Tareas teóricas

- Cada una de ellas tienen una incidencia en las notas alcanzadas por los estudiantes, el uso independiente de cada una de ellas no aporta en una formación integral y comprensiva de los conocimientos teóricos en los procesos prácticos y viceversa.

- Cada factor cumple con un aporte significativo, medida que el ciclo va completándose, los resultados mejoran en el estudiante, consiguiéndose así conocimientos teóricos - prácticos dentro de niveles aceptables, necesarios para avanzar en su formación académica.

- Los factores metodológicos de enseñanza como variables de investigación tienen dependencia sobre las variables dependientes (notas alcanzadas por los estudiantes).

- Los valores alcanzados por los estudiantes en las evaluaciones mantienen menos dispersión a medida que se va completando el ciclo de enseñanza planteada para las materias citadas.

\section{Referencias bibliográficas.}

Gonzales, N. Y García, M. (2007). El aprendizaje cooperativo como estrategia de enseñanza - aprendizaje en psicopedagogía (UC): repercusiones y valoraciones de los estudiantes. Revista Iberoamericana de educación (ISSN: 1681-5653). Consultado en febrero 2021. Obtenido de: http://www.ardilladigital.com/DOCUMENTOS/EDUCACION\%20ESPECIAL/ APRENDIZAJE\%20COOPERATIVO/Apredizaje $\%$ 20cooperativo\%20como\%2 0estartegia\%20de\%20E-A\%20-\%20Gonzalez\%20y\%20Garcia\%20-\%20art.pdf

Lira, R. (2010). Las metodologías activas y el foro presencial: Su contribución al desarrollo del pensamiento crítico. Revista Actualidades investigativas en educación, 4. Obtenido de: https://www.redalyc.org/pdf/447/44713068008.pdf 
Ibarrola - García, Sara. (2013). El conocimiento práctico del profesor: ¿Cuándo empieza todo? REDU Revista de docencia universitaria, pp. 219.

Rodríguez, J. y Alamilla, P. (2018). La complejidad del conocimiento profesional docente y la formación del conocimiento práctico del profesorado. Actualidades investigativas de educación, vol. 18, núm. 2, pp. 1-24. Obtenido de: https://www.redalyc.org/jatsRepo/447/44758022017/html/index.html

Barros, Brenda; Chavarría, Margarita y Paredes, Joaquín (2008). Para analizar la transformación con tic de la enseñanza universitaria. Un estudio exploratorio sobre creencias pedagógicas y prácticas de enseñanza con tic en universidades latinoamericanas. REIFOP, 11. Obtenido de http://www.aufop.com/aufop/home/

Delors Jacques (1996). La Educación encierra un tesoro, informe a la UNESCO de la Comisión Internacional sobre la Educación para el Siglo XXI (compendio), pp. 8. Obtenido de https://unesdoc.unesco.org/ark:/48223/pf0000109590_spa

Cano García, Ma. Elena (2008). La evaluación por competencia en la educación superior. Profesorado. Revista de Curricular y Formación de Profesorado. vol. 12, núm. 3, pp. 1-16. Universidad de Granada. Granada. Obtenida de: https://www.redalyc.org/pdf/567/56712875011.pdf

Registro Oficial Suplemento 298 de 12-oct.-2010 Ultima modificación: 02-ago.-2018. Ley orgánica de Educación Superior, LOES. Obtenido de: https://www.epn.edu.ec/wp-content/uploads/2018/08/LOES.pdf

Garduño, L. (1999). Hacia un modelo de la evaluación de la calidad de instituciones de educación superior. Revista Iberoamericana de educación. Núm. 21, pp 93-103. Universidad Siglo XXI. Obtenido de: https://rieoei.org/historico/documentos/rie21a06.PDF

Himmel, E. (2001). La evaluación de aprendizajes en la educación superior. Calidad en la educación, (15), pp. 1-8. Obtenido de https://doi.org/10.31619/caledu.n15.447

Romero, O. Amaya, R. y Castaño, S. (2020). Recurso didáctico para la enseñanza en el programa de tecnología en Gastronomía en la Universitaria Uniagustiniana, pp. 3$10 . \quad$ Obtenido de http://repositorio.uniagustiniana.edu.co/bitstream/handle/123456789/1455/Rome roArdila-OscarHernando-2020.pdf?sequence=1\&isAllowed=y

Roig, R. Lledó, A. Antolí, J, y Pellín, N. (2019). Redes de Investigación e Innovación en Docencia Universitaria. Volumen 2019, pp. 541 - 553. Obtenido de 
https://rua.ua.es/dspace/bitstream/10045/99176/1/Redes-InvestigacionInnovacion-Docencia-Universitaria-2019-48.pdf

Correia G. Araújo D. Fernández L. Leão P. y Pinheiro P. (2012). GESTIÓN DE CALIDAD DEL SERVICIO DE ALIMENTOS Y BEBIDAS. La importancia del manipulador de alimentos en la calidad del servicio hotelero de la ciudad de Joao Pessoa, Brasil Estudios y Perspectivas en Turismo, vol. 21, núm. 3, pp. 763-777.

Cifuentes, I. (2012). Guía metodológica del curso gastronomía guatemalteca por regiones de la escuela taller de hotelería zunil para el fortalecimiento de la identidad nacional y la virtud del patriotismo (Tesis de Licenciatura). Universidad del Istmo. Facultad de educación, Guatemala.

Castillo, M. (2012). Mejoramiento pedagógico en el ámbito universitario (Tesis de maestría). Universidad de la Sabana. Facultad de educación. Colombia.

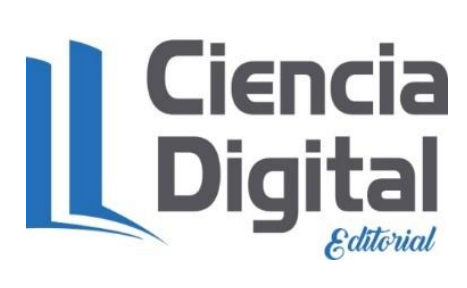




\section{PARA CITAR EL ARTÍCULO INDEXADO.}

Guambi Espinosa, D. R., Marín Parra, I. M., \& Ávalos Pérez, M. C. (2021). Análisis del modelo docente universitario y el uso de metodologías de enseñanza, aprendizaje en la evaluación de asignaturas de la praxis profesional en carreras de Gastronomía. ConcienciaDigital, $\quad$ 4(1.2), 235-255. https://doi.org/10.33262/concienciadigital.v4i1.2.1591

\section{Ciencia \\ Ligital}

El artículo que se publica es de exclusiva responsabilidad de los autores y no necesariamente reflejan el pensamiento de la Revista Conciencia Digital.

El artículo queda en propiedad de la revista y, por tanto, su publicación parcial y/o total en otro medio tiene que ser autorizado por el director de la Revista Conciencia Digital.

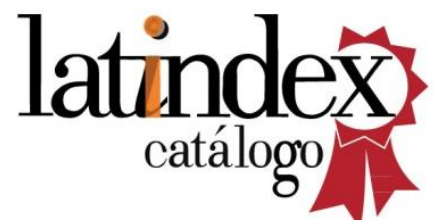

